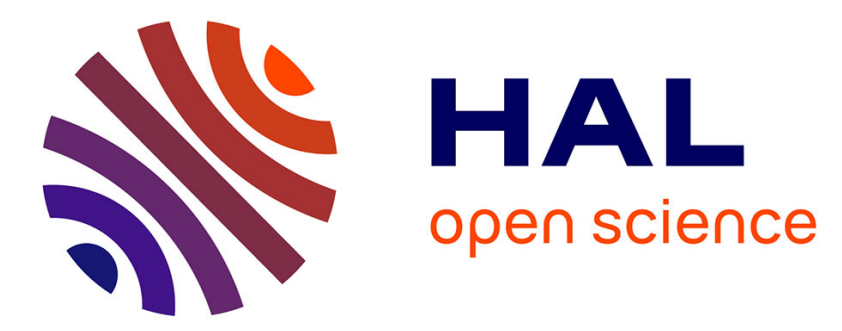

\title{
L'enseignement du littéraire dans le secondaire algérien à l'ère du numérique
}

\author{
Abdelkader Benali
}

\section{To cite this version:}

Abdelkader Benali. L'enseignement du littéraire dans le secondaire algérien à l'ère du numérique. Le Français Aujourd'hui, 2012, 178, pp.115 - 132. halshs-00919797

\section{HAL Id: halshs-00919797 https://shs.hal.science/halshs-00919797}

Submitted on 18 Dec 2013

HAL is a multi-disciplinary open access archive for the deposit and dissemination of scientific research documents, whether they are published or not. The documents may come from teaching and research institutions in France or abroad, or from public or private research centers.
L'archive ouverte pluridisciplinaire HAL, est destinée au dépôt et à la diffusion de documents scientifiques de niveau recherche, publiés ou non, émanant des établissements d'enseignement et de recherche français ou étrangers, des laboratoires publics ou privés. 


\title{
L'ENSEIGNEMENT DU LITTÉRAIRE DANS LE SECONDAIRE ALGÉRIEN À L'ÈRE DU NUMÉRIQUE
}

\author{
Abdelkader BENALI \\ Université Paris Ouest Nanterre La Défense \\ MoDyCo - UMR 7114
}

\begin{abstract}
J'ai toujours résisté à ces dilemmes imposés et refusé les exclusions mutuelles qui semblaient fatales à la plupart de mes contemporains. L'étude littéraire doit et peut réparer la cassure de la forme et du sens, l'inimitié factice de la poétique et des humanités.
\end{abstract}

A. Compagnon, La Littérature pour quoi faire ?, 2007.

Leibniz voulait développer des boussoles du savoir ; il désirait généraliser les cartes et les répertoires de contenus. Il voulait faire l'inventaire du trésor public (le « thésaurus ») des savoirs disponibles, créer une immense mémoire virtuelle de la culture et des arts. Tablant sur cette richesse, il voulait en profiter pour réarticuler les notions, pour construire des rangements en étoile, aménager des carrefours, multiplier les raccordements réticulaires, visualiser les relations entre les informations par des tableaux de correspondances. Il voulait que le transversal l'emporte sur le sectoriel, que les liens horizontaux ou diagonaux réunissent ce que l'on croyait disjoint. Immense rêve panoptique, synoptique, combinatoire. Il voulait substituer à la multiplicité asservissante une représentation plus compacte. Il pensait que la véritable science est source de simplification, et qu'elle s'abrège en s'augmentant.

À l'ère des réseaux et du numérique, l'idée fondamentale de Leibniz parait plus actuelle que jamais. Son rêve prend une actualité brulante, au moment où se dessinent les "sociétés du savoir » et où l'on s'interroge sur leur orientation, en particulier sur les nécessaires réformes de l'éducation, vivier central, stratégique de nos sociétés futures (Quéau 2010).

L'introduction des technologies de l'information et de la communication dans le programme du secondaire algérien représente une restructuration globale de la pédagogie et des programmes qui est instituée afin d'améliorer la qualité des apprentissages et faire progresser la réorganisation de l'enseignement. L'ensemble de ces variables étant constamment interrogées à l'intérieur d'une nouvelle vision de l'acte de formation dans lequel «l'approche par la compétence » fonde la matrice de tout le système.

Dans le nouveau programme du secondaire algérien, initié en 2005, l'introduction du texte littéraire sert un double objectif : il peut être utilisé à des fins linguistiques, c'est un moyen d'apprentissage par lequel les élèves peuvent explorer tous les possibles (phonétiques, graphiques, morphosyntaxiques, sémantiques) ; il peut aussi être utilisé comme moyen et dispositif pédagogique pouvant assurer une médiation avec la culture étrangère ou l'intraculture. Découvrir l'autre, ce n'est pas seulement apprendre comment il vit, c'est aussi mesurer les différences qui peuvent exister entre deux façons d'envisager le monde, afin de les relativiser. Le manuel porte cette relativisation de manière implicite, en proposant des réflexions qui tendent à éviter tout jugement trop hâtif. 
C'est dans cette optique que nous envisageons d'analyser et de détailler l'ensemble des moyens proposés pour l'enseignement du texte littéraire face aux nouvelles facettes du réseau numérique. Cette situation nous conduit à nous interroger sur l'apprentissage de ce type de texte où l'implication de la production de sens et la saisie de la signifiance sont de mise. Quelle langue abordée, celle du numérique ou celle du littéraire ? L'enjeu est certainement de taille, surtout si le texte se prête à plusieurs lectures lorsqu'il est présenté dans son contexte. Par texte, il nous faudra faire la distinction entre texte numérisé ou texte du livre papier.

Notre article présente un corpus (composé dans des classes de lettres du secondaire) qui nous ont permis d'analyser les pratiques pédagogiques, les enjeux didactiques et pédagogiques, ainsi que les activités orales et écrites que peut présenter l'enseignement du texte littéraire lié aux technologies numériques.

\section{Introduction des nouvelles technologies de l'information et de la communication en Algérie}

Face à un contexte international en perpétuel changement et face à des enjeux internationaux décisifs, auxquels elle ne peut se montrer indifférente, l'Algérie a amorcé l'intégration des nouvelles technologies de l'information et de la communication (dorénavant NTIC) dans plusieurs secteurs de l'appareil de l'état, entre autre dans celui de l'éducation. Ainsi, suite à des décisions politiques, et tout comme ses pays voisins, un programme national d'introduction des NTIC et de développement dans l'enseignement a été mis en place et un certain nombre de mesures ont été prises par les pouvoirs publics. Pour entamer immédiatement la procédure, le président algérien a ordonné l'affectation d'un budget sur quatre années (2001-2004) qui s'élève à près de trois milliards de dinars algériens, pris sur le programme de soutien à la relance économique et destinés à engager la mise en œuvre. Ensuite, d'autres réalisations ont eu lieu. En effet, l'état algérien a commencé par installer une commission nationale des programmes baptisée $\mathrm{CNP}$, dont la mission est la refonte des contenus d'enseignement avec, notamment, l'intégration de la dimension technologique dans les disciplines et les activités pédagogiques. Des réformes sont ainsi réalisées, portant sur l'introduction et le développement des utilisations des NTIC dans l'enseignement. En même temps, et pour activer le processus de l'innovation, le ministère de l'éducation national algérien (MEN) a créé un Centre national de l'innovation pédagogique et des TICE (CNIPTICE) chargé de la gestion et de la mise en œuvre du programme NTIC pour l'enseignement primaire et secondaire, et de développer les usages au plan pédagogique et administratif à l'intérieur des établissements scolaires, et même de prévoir des mises à niveau pour les personnels de l'éducation. En outre, le MEN a entrepris la mise en place des équipements. De ce fait, 1300 lycées, 3500 collèges et 8000 écoles primaires constituent le nombre total des institutions scolaires algériennes ainsi dotées, à raison de quinze ordinateurs par établissement, complétés d'autant de périphériques, reliés en réseau et connectés à internet. Dix de ces machines sont destinés aux élèves et cinq sont mis à la disposition des enseignants. Le ministère lui-même, plus huit établissements sous sa tutelle, les sept instituts de formation et les quarante-huit délégations ont aussi pu bénéficier d'une infrastructure du même type, assurant ainsi la mise en réseau des différentes directions.

Pour inciter le recours à ces équipements et familiariser les enseignants à l'utilisation de ces outils, des sessions massives de formation ont été engagées par le MEN. Il a également encouragé les inspecteurs, les professeurs d'enseignement secondaire et ceux du cycle moyen à y participer. Pour généraliser l'introduction des NTIC dans l'enseignement, le MEN a, en plus, établi un plan 
de développement du secteur de l'éducation pour la période de 2005-2009 avec trois mille laboratoires informatiques à acquérir par les collèges, ainsi qu'une plateforme et des sites Webs à développer et à mettre en place pour amorcer l'enseignement à distance. Pour suivre ce grand projet d'introduction des NTIC dans l'enseignement et impulser des usages plus systématiques de l'ordinateur, des séminaires internationaux se sont tenus; d'abord à Paris le 28 mai en 2004, sous l'égide de l'université de Strasbourg, mais à l'initiative des étudiants de la promotion de janvier 2003 du DESS - UTICEF (utilisation des technologies de l'information et de la communication dans l'enseignement et la formation) ; ensuite à Alger en novembre 2005 sous le titre suivant : « L'usage des NTIC pour l'enseignement, la formation et l'apprentissage : retour d'expériences ». Depuis 2003, l'Algérie sollicite en effet des coopérations internationales, car le gouvernement est conscient de l'ampleur du projet et du fossé numérique qui reste à combler. C'est ainsi que des missions d'expertise ont été réalisées en partenariat avec la France, afin d'aider à l'installation et au développement des compétences en matière d'intégration pédagogique des NTIC. Des appels destinés aux bailleurs de fonds ont ainsi été lancés afin de continuer à élargir et de maintenir ces infrastructures dans les différents secteurs de l'éducation.

\section{L'introduction du littéraire dans les nouveaux programmes}

Dans le nouveau programme du secondaire algérien, initié en 2005, l'introduction du texte littéraire sert un double objectif : il peut être utilisé à des fins linguistiques ; c'est un moyen d'apprentissage par lequel les élèves peuvent explorer tous les possibles (phonétiques, graphiques, morphosyntaxiques, sémantiques). Mais il peut aussi être utilisé comme moyen et dispositif pédagogique pouvant assurer une médiation avec la culture étrangère ou intraculture. Découvrir l'autre, ce n'est pas seulement apprendre comment il vit, c'est aussi mesurer les différences qui peuvent exister entre deux façons d'envisager le monde, afin de les relativiser. Le manuel porte cette relativisation de manière implicite, en proposant des réflexions qui tendent à éviter tout jugement trop hâtif.

En effet, la polysémie du texte littéraire permet aux élèves de se distancier, de se méfier des évidences ; L. Porcher et M. Abdallah-Pretceille (1996) considèrent la littérature comme « un lieu emblématique de l'interculturel », du fait de ses caractéristiques intrinsèques. De façon générale, la littérature permet une confrontation des élèves avec l'altérité et avec une autre perception du monde. Comme le confirment les deux auteurs « Le texte littéraire, production de l'imaginaire, représente un genre inépuisable pour l'exercice artificiel de la rencontre avec l'Autre : rencontre par procuration certes, mais rencontre tout de même ».

Le retour du texte littéraire en tant que témoignage culturel est ainsi une des formes possibles que les concepteurs appellent de leurs vœux et à laquelle se joignent les innovations portant sur les différentes activités. Cet apprentissage implique que la production de sens et la saisie de la signifiance passent forcément par l'utilisation de la langue. La pratique est alors plus aisée dans le cas où le texte se prête à plusieurs lectures lorsqu'il est présenté dans son contexte.

Le texte littéraire va se présenter comme un ensemble situant une information dans le temps et dans l'espace, cette information est en relation avec une culture ou une idéologie. Il est le lieu favorable où s'exercent le contact et l'apprentissage de la langue et la culture à travers une interaction avec le texte.

Les relations de la culture, de l'intraculture et de l'interculturel sont fortement présentées dans les programmes et véhiculées par des supports insérés dans le manuel : «La familiarisation avec 
d'autres cultures francophones pour comprendre les dimensions universelles que chaque culture porte en elle ».

Le nouveau programme poursuit des objectifs qui dépassent le cadre de la classification typologique des textes. Il s'inscrit dans une logique de projet pédagogique et vise l'installation de compétences par des activités variées qui permettent aux élèves de s'approprier les règles régissant les différents discours qui les sous-tendent. Ce nouveau programme met aussi l'accent sur l'utilisation des NTIC pour l'enseignement de ces textes en s'attelant à ce que l'apprentissage permet : le développement des compétences en matière de nouvelles technologies, d'une part, et la compréhension des textes littéraires, d'autre part, sur des supports autres que le papier.

\section{Impact des NTIC sur l'enseignement du littéraire}

\section{Découverte scientifique et diversité des échanges}

L'internet a apporté une certaine dimension communicative à l'activité pédagogique et a permis de renforcer le travail collaboratif. Cette propriété, qui avait déjà été identifiée par F. Chacón en 1992, est aujourd'hui décuplée par l'usage généralisé de trois grands types d'outils :

- les forums et listes de diffusion qui permettent aux élèves de partager de l'information;

- les wikis pour la publication de contenus (voir Wikipédia, l'encyclopédie collaborative);

- les weblogs qui permettent la présentation de données personnelles et qui inaugurent les eportfolio particulièrement utiles pour l'évaluation.

Ensuite, ces usages nouveaux s'accompagnent d'une possibilité d'ouverture de l'école sur la société :

- Internet ouvre l'accès à une base d'information plus large sur tous les sujets abordés en éducation. La plupart des médias offrent maintenant une version en postcast (diffusion différée) de leurs émissions. Une émission de radio (voire de télévision) peut être consultée sur un lecteur MP3 ou transportée sur une clé USB.

- Possibilité d'action réelle : enquêtes sur internet, échanges de travaux avec d'autres classes...

- Possibilité de publication et de valorisation des travaux : les sites d'établissement se couplent avec des sites thématiques (environnement, géographie, histoire, etc.) présentant les travaux des élèves comme sources d'information et documents de travail pour d'autres. Cette diversité apparait très bien dans la classification de modèles pédagogiques. À vrai dire, les usages pédagogiques sont quasiment infinis.

L'internet a également apporté ce qui aura probablement encore plus d'impact sur les mutations de l'Éducation dans de nombreux pays : la possibilité de relier l'ensemble des acteurs entre eux, de mutualiser et de capitaliser de manière instantanée et efficace les efforts pédagogiques, que ces efforts portent sur l'application des NTIC ou sur tout autre sujet. En résumé, on peut affirmer qu'internet a permis aux NTIC de sortir du monde des spécialistes pour s'intégrer à l'ensemble des activités éducatives.

\section{De nouvelles modalités d'apprentissage}

Dans le contexte algérien, l'enseignement des textes littéraires semble plus délicat si nous ne prenons pas en compte les variations culturelles immédiates et originales du texte. Dans la phase d’imprégnation, le professeur doit ainsi initier les élèves au contexte socioculturel et économique 
de l'époque, celle qui correspond à l'édition-production du texte. Nous pensons ainsi que le rapport au texte peut être préparé et précédé par un résumé portant, entre autres, sur les circonstances durant lesquelles le texte a été écrit. Il nous semble dès lors indispensable de procéder à l'enseignement d'une langue - et donc d'une culture littéraire - en prenant en considération ces aspects socioculturels et économiques.

De ce point de vue, en tant qu'outil pédagogique, les NTIC peuvent renforcer l'innovation dans l'enseignement et la motivation dans l'apprentissage, permettant une éducation où chaque élève peut en quelque sorte apprendre à son rythme. En d'autres termes, il s'agira de rendre l'individu apte à « apprendre à apprendre » et à « apprendre à agir avec le monde entier».

Dans ce contexte, les textes littéraires soumis aux nouvelles technologies, notamment par les supports numérisés, permettent l'émergence de nouvelles perspectives de lecture et de compréhension. La tâche dévolue à l'enseignant change constamment, car les NTIC agissent relativement :

$[\ldots]$ au gré des transformations des systèmes éducatifs aussi bien que du métier et des conditions de travail des enseignants. [...] Ni la vidéo, ni l'ordinateur, ni le multimédia n'ont, à ce jour, bouleversé le métier d'enseignant. [...] Si de nouvelles compétences surgissent, ce n'est donc pas pour répondre à de nouvelles possibilités techniques, mais parce que la vision ou les conditions d'exercice du métier se transforment (Perrenoud 2001).

\section{Accessibilité par l'image}

Sur un autre plan également lié aux apprentissages, la lecture par l'image retient l'attention des élèves et permet une compréhension approfondie, même si elle est soumise à des variables d'interprétation plus nombreuses. Si cette lecture électronique semble être le résultat d'une somme de phénomènes inconscients, dominés par la discontinuité, elle ne correspond guère à la représentation que nous nous faisons des actes traditionnels de lecture rapide ou approfondie ; il s'agit plutôt d'une lecture particulière, diagonale, lente (Nielson et Loranger 2008).

Ainsi, nous pouvons affirmer que l'effort le plus important est de :

1. rendre le texte littéraire plus accessible à l'étude, tout en ayant plus d'efficacité dans les travaux de groupe ;

2. mettre en évidence les principes de l'approche communicative ;

3. fournir un modèle de réussite pour la compréhension des textes littéraires ;

4. surmonter la lecture des textes difficiles, par des ressources et des moyens informatiques qui cherchent à faciliter leur accès, notamment via les images.

Pour cela, on mesure qu'une tension, féconde en elle-même, a toujours prévalu dans les réflexions sur l'enseignement des lettres et notamment sur l'explication de texte littéraire. Tension entre une approche plus soucieuse de "poétique » au sens rhétorique du mot, et une tradition plus sensible aux « humanités ». Cette démarche d'enseignement du littéraire, en tenant compte des NTIC, donne à réfléchir sur le contexte et les contenus dans lesquels se développent les méthodes d'apprentissage. 


\section{Quels enjeux et apports didactiques?}

Les NTIC permettent la découverte de nombreux phénomènes littéraires, sous différents angles : esthétiques, poétiques, linguistiques etc. Le traitement de l'information ne s'égare nullement de la trajectoire conçue par le texte en mouvement. Cette capacité donne aux élèves la possibilité de découvrir sa portée littéraire par des données réelles, présentées sous forme de figures simples à interpréter. Mais elles ont aussi d'autres intérêts et d'autres fonctions.

\section{Développer l'esprit critique}

Le texte littéraire peut engager une multitude de lectures allant de la compréhension littérale à la compréhension inférentielle ; c'est pourquoi il est nécessaire de présenter toutes les dimensions herméneutiques qu'offre le texte. Les NTIC facilitent la production de ces représentations formulées par l'œuvre. Dans ce contexte, les élèves aiguisent leur esprit critique à l'égard du texte présenté à l'étude. Ils peuvent également recourir aux informations qui leur sont utiles pour résoudre les différentes composantes du sens et l'efficience de la signification.

\section{Comprendre et s'ouvrir à l'autre}

L'environnement numérique a le privilège de faciliter l'accès à l'information et par conséquent un excellent moyen d'entrer en contact avec les autres. Les textes littéraires sont forcément liés à une civilisation et donc porteurs de valeurs sociétales. Cette connaissance révélée aux jeunes lecteurs implique une interaction sans limite avec les textes, leur permettant de découvrir l'autre et de bâtir des relations virtuelles qui s'organisent à travers les phrases et la philosophie, une langue et une épistémologie, engageant une réelle découverte de l'autre. Ce partage de connaissance met en évidence les liens qui unissent la littéralité des œuvres à leurs auteurs et à leurs lecteurs.

\section{Approcher la littéralité des œuvres}

À la différence de beaucoup de textes que sont l'explicatif, l'argumentatif et le descriptif, les textes littéraires ont la particularité d'engager les jeunes lecteurs dans une pluralité de sens, une multitude d'interprétations liées à l'essence même de l'œuvre. Cette distinction engendre ce qu'on appelle plus communément les non-dits ou les sous-entendus, qualités intrinsèques des textes littéraires. C'est ce qui donne le plaisir de leur lecture. L'implicite y est souvent présent et le lecteur a le privilège de le comprendre selon une diversité de moyens.

C'est dans ce sens que, dans son intervention, P. Laudet insistait en juin 2011 sur certains aspects de la compréhension des textes littéraires, aspects qui relèvent souvent de l'implicite pour beaucoup d'œuvres. Il souligna à cet effet :

Entendre les textes bien sûr, mais aussi les sous-entendre. Sous bien des aspects, le texte littéraire est toujours plus ou moins allusif. C'est bien dans l'exercice de l'explication de texte que se construit l'accès des intelligences au « second degré », à l'implicite, à l'humour... La compétence visée, c'est bien de permettre aux élèves, s'agissant des énoncés, d'entendre ce qui se dit dans ce qui est dit, et de se convaincre, il y va de leur liberté, qu'il y a toujours plus à entendre dans ce qui est dit que ce que ce dire même prétend signifier. Comme le dit Novarina, descendre (ou monter !) dans le langage pour en savoir plus.

De plus, cette vivifiante réévaluation incessante du sens à partir d'une situation de perte de sens permet la « lecture aux éclats», une lecture qui « égare », entraine dans des incertitudes aux lieux et à la place de la positivité promise ou escomptée. 
La compréhension littéraire est donc bien une lecture au second degré, assumée, qui autorise le jeu libre avec les signifiants et l'écoute fine et futée des significations. Elle ne redoute pas les approches paradoxales. Elle combat l'esprit de sérieux et les dogmatismes qui vont avec. Elle sait « jouer» du texte, comme d'une balle ou d'un violon, et se joue de lui. Elle sait en déjouer les attendus et en rejouer les contenus.

\section{Accéder à l'interprétation littéraire}

Il est impossible de tout dire des textes et de leur visée. U. Eco a réfléchi à ce risque permanent qui serait de manquer l'altérité véritable du texte par l'excès mal contenu d'une interprétation pouvant aller jusqu'à la dissipation de l'œuvre originelle, devenue le miroir servile de l'ego du commentateur. Si le texte reflète l'idée de son auteur, le lecteur examine cette possibilité par une relative interprétation, tant les possibilités du texte s'offrent à la nature du hasard et au désir du lecteur de se construire une représentation conforme à sa compréhension.

L'herméneutique favorise une démarche qui éviterait de sombrer dans l'introduction d'une gnose réservée aux seuls initiés capables de présenter une rigoureuse interprétation des textes.

Un texte ne peut aller au-delà de ses limites et on ne peut lui créditer d'autres fins, seule notre crédibilité lui assure sa valeur significative. À travers les textes que nous lisons et que nous observons par les images - entre autres avec les textes numérisés -, nous cherchons souvent le motif ayant servi au tissage de l'énigme, au paradoxe et à la fantaisie textuelle. Cet état de fait n'est pas perceptible à première vue ou lors de la première lecture ; il faut une sorte de vigilance et de concentration, de lire au-dedans, entre les lignes. Entendre finement ce qui se dit dans ce qui est dit, ne pas être dupe de la lettre et avoir un peu d'esprit! Mais les psychanalystes le savent bien, il suffit finalement pour cela de ne rien faire qu'écouter ce qui est dit, ostensiblement. Ne pas chercher l'esprit ailleurs que dans la lettre, qui le manifeste toujours. C'est alors La Lettre volée d'E. Poe qui nous apprend à ne pas manquer le signifiant en évidence sur la cheminée, quand tous l'ont cherché.

Les images des textes littéraires sont conçues dans le cadre d'une mise au point reflétant, ne serait-ce que d'une manière relative et très rapprochée du contexte textuel, l'interprétation plausible et acceptable des limites significatives de ces textes.

\section{Saisir l'authenticité des textes}

Dans l'approche communicative qui, depuis le début, a mis l'accent sur le document authentique, le texte littéraire a paradoxalement été négligé. Pourtant, dès les premières années qui suivent l'indépendance de l'Algérie, le texte littéraire a toujours été aux premières lignes des différents programmes. Aujourd'hui, nous assistons à une évolution de la part des concepteurs de ces programmes, qui affirment l'importance des textes littéraires car ils représentent la face essentielle des textes authentiques.

Cet engouement est dicté par l'idée qu'une langue « authentique » doit être portée par des documents et des situations également authentiques. Les textes littéraires ont ce privilège d'être des images de faits et d'évènements d'un quotidien que les élèves peuvent apprécier et comprendre. Ils constituent des supports efficaces qui dégagent des pistes d'apprentissage facilement préhensibles.

Les textes littéraires ont l'avantage d'être utilisés dans une situation se rapprochant de ce qu'explore le lecteur comme possibilité d'interprétation ; cette possibilité lui confère l'avantage d'un document qui contient, dans sa propre écriture, les moyens de sa compréhension. Pour 
n'importe quel lecteur un texte littéraire est un document qui a un contexte sociohistorique et des références culturelles. Le texte fournit lui-même au lecteur les indices nécessaires à sa compréhension, et les différentes valeurs culturelles qu'il dégage s'inscrivent dans un contexte précis où il n'est nullement aberrant de faire une fausse route à partir d'une information décontextualisée.

\section{Accroitre les interactions}

Nous l'avons vu, la compréhension des textes ne relève pas uniquement des textes eux-mêmes, mais des possibles ouverts au lecteur qui, à partir d'indices spécifiques, peut en tirer sa propre interprétation, mais une interprétation inscrite sur ses connaissances du monde. Cette façon de concevoir la compréhension écrite ouvre la voie à l'étude des textes littéraires puisqu'on accepte que le lecteur étranger puisse jouir d'une certaine liberté dans la construction du sens de ces textes.

Outre le fait d'être un document authentique inestimable, le texte littéraire attire l'attention des élèves, il les fascine à cause de certains traits qui lui sont particuliers. "Toute l'attention est sollicitée, dès l'approche, non par les informations dont le texte peut être porteur mais par le texte lui-même dans sa texture visuelle, sonore, grammaticale et sémantique. Il y a là des caractéristiques propres aux documents littéraires qui les adaptent particulièrement à leurs inévitables conditions de réception dans une classe de langue étrangère » (Besse 1982 : 25). Parmi ces caractéristiques, $\mathrm{H}$. Besse mentionne la disposition typographique particulière, le rythme, les correspondances internes de forme ou de sens, les ambigüités d'ordre syntaxique qui sont « autant d'indices par lesquels le document littéraire exerce sur le lecteur son insolite fascination » (Ibid. : 26).

\section{Développer les savoir-faire linguistiques, sociohistoriques et stylistiques}

Linguistiques : Le texte littéraire est de prime abord un texte où s'exerce la langue dans toutes ses dimensions. Quand on s'intéresse à la littérature, on ne se contente pas de travailler des notions élémentaires de la langue, bien au contraire ; il faut établir les relations qu'entretient la langue avec l'univers du texte sur le plan du lexique, de la sémantique et de la continuité discursive. La langue garantit la validité du sens ; elle définit ainsi l'univers représenté dans des perspectives qui définissent les normes liées aux différents paramètres de la communication.

Ce que le texte avance pour justifier sa légitimité littéraire, il le doit à la langue employée qui permet de faire émerger un discours réussi et compréhensif. Cette "représentativité » du texte littéraire trouve son terrain de prédilection dans l'utilisation des différents supports numériques avec lesquelles les élèves découvrent les subtilités de la langue par des éclaircissements terminologiques, des définitions proprement linguistiques et des remarques substantielles sur les registres de langue exprimés par les textes littéraires introduits dans le programme scolaire du secondaire algérien.

Sociohistoriques : Le texte littéraire représente le monde et la société dans lesquels évolue l'histoire ; ce qui évoque les circonstances traitées dans un cadre bien précis et distingue fondamentalement les propriétés et les caractéristiques d'une société. Les objets et les personnages évoqués dans les textes littéraires sont le reflet de mouvements philosophiques, le symbole d'une classe et la représentation d'une instance à part et sans égal. Il n'y a donc pas d'œuvre littéraire qui ne caractériserait pas une société par référence à une autre. La diversité des 
sociétés implique différents niveaux d'évolution, différents discours, différentes philosophies et une multitude d'affirmations d'univers narratifs.

Face aux moyens numériques, les textes littéraires engagent une action où se mêlent des spécificités liées à la métamorphose des sociétés contemporaines. Les élèves se réjouissent de la découverte de ces NTIC qui recourent à des images en mouvement, en parcourant différents paysages sociohistoriques ayant contribué à la plénitude des textes littéraires.

Stylistiques : L'approche stylistique entend faciliter l'interprétation des textes en s'appuyant sur une étude des faits de langue caractéristiques du "style » d'un écrivain ou d'un ensemble d'écrivains (Maingueneau 2003 : 3). Cette stylistique, qui combine plusieurs procédés d'expression, met en valeur l'esthétique du texte afin de donner un nouvel éclairage pour son approche et son étude. La langue est associée à ces procédés rhétoriques qui portent à la connaissance des jeunes lecteurs des matériaux sémiotiques pouvant investir leur imaginaire. Dans le texte littéraire, les règles qui gouvernent son esthétique sont souvent d'ordres tacites, implicites ou sous-entendus. Dès lors, aborder stylistiquement les textes littéraires, c'est privilégier des figures qui établissent des liens étroits avec la représentation de l'univers textuel évoqué. Dans ce sens, D. Maingueneau souligne que :

[...] Toute œuvre qui figure au corpus de la littérature pousse son lecteur à traquer l'implicite. Il y a même des œuvres qui se donnent comme "allégoriques ", "symboliques ", " métaphoriques » [...], c'est-à-dire qui indiquent nettement au lecteur qu'il lui faut traquer l'implicite. (1990: 78)

Cette stylistique émerge d'une manière subtile dans le monde du numérique ; elle pose des limites à l'interprétation et donc à l'imaginaire parfois débordant des élèves ; elle lui assure en retour un crédit de compréhension et des capacités d’interprétation souvent insoupçonnées. Les séquences présentées dans l'œuvre littéraire fournissent, grâce aux images en mouvement, un décor qui définit les zones de la valeur des mots et du texte par une singularité incomparable.

\section{Analyse d'une séquence didactique associant littérature et NTIC}

\section{Objectifs et attendus}

Pour analyser la pertinence et l'efficacité du dispositif didactique envisagé, nous avons conçu une séquence avec un protocole précis, permettant d'apprécier les effets de l'enseignement des textes littéraires dans toutes les dimensions et perspectives décrites supra.

La séquence expérimentale s'est déroulée dans le contexte scolaire particulier de la société algérienne, avec ses caractéristiques et ses finalités propres, déterminées par les missions affectées institutionnellement à l'école. Cette séquence a été mise en place dans un établissement public, il s'agit du lycée Hadj-Aissa Aboubakeur, situé à Laghouat (sud de l'Algérie), durant l'année scolaire 2009-2010. Le public est constitué d'élèves d'une classe de troisième année du secondaire, série « lettres », comprenant 21 filles et 19 garçons, soit un total de 40 élèves. L'âge de ces élèves varie entre 17 et 19 ans. Ils ont suivi une scolarité régulière et n'ont pas redoublé durant leur cursus scolaire antérieur, cependant leurs performances scolaires restaient hétérogènes. Le choix de ce niveau de classe n'est pas anodin puisqu'il cadre avec le programme étudié durant les deux dernières années, un programme qui s'actualise en troisième année avec la découverte de nouvelles spécificités du texte littéraire.

Dans ce cadre, nous nous sommes attachés à comprendre comment ces lycéens appréhendent, analysent et restituent les textes littéraires. Il s'agissait d'observer les différents procédés exposés 
précédemment pour la compréhension de ces textes dans un contexte où se mêlent technologie et littéracie.

Ce choix a également été motivé par le fait que le corpus de textes proposé - à valeur essentiellement poétique - répond à nos attentes et valide ainsi son accessibilité par les élèves. Il est important de considérer que la démarche reste expérimentale, par l'objectivation de nos hypothèses didactiques et la définition d'un cadrage théorique précis. L'analyse présentée ici reste donc essentiellement didactique et peut être considérée comme un rapport de recherche en action. Il s'agit cependant d'actions spécifiques liées à l'étude de textes littéraires particuliers, mais aussi une étude soumise à des interactions didactiques régies par des procédures et des règles définies. Ces actions visent à construire une compréhension textuelle par le biais d'images en mouvement où les élèves, sans lire le texte, procèdent à un codage linguistique en français.

La séquence didactique se répartit en trois séances, correspondant aux éléments clés de l'étude du texte littéraire choisi, dans un contexte impliquant le recours aux NTIC. Chaque séance correspond à une activité de compréhension du texte en lien avec l'une de ses caractéristiques.

Pour les besoins du présent article, nous avons ciblé les séances et le support permettant d'identifier la compréhension des enchainements caractéristiques d'un texte littéraire ${ }^{1}$ présenté dans un univers numérique spécifique, en l'occurrence à travers la description des images associées et leur analyse fonctionnelle. Cette séance s'attache également à repérer les variables et constantes rédactionnelles des élèves et la manière dont ils sont parvenus à les maitriser dans un réseau numérique. Les écrits produits constituent ainsi une activité dans laquelle les élèves sont amenés à jouer avec des contraintes psycholinguistiques fortes, mais aussi avec des variantes linguistiques souvent inégalement maitrisées et donc difficilement hiérarchisables - sans compter les écarts culturels et sociétaux évoqués plus haut.

La tâche proposée consiste à comprendre et à analyser le texte littéraire soumis sous la forme d'images et à en proposer une version écrite en vers dans une forme linguistique assurant sa compréhension. Sans lire le poème, les élèves doivent observer les images en mouvement et proposer dans un texte versifié ce qu'ils aperçoivent sur l'écran. Les images correspondent, par le biais d'un logiciel, au poème proposé, certaines descriptions, sauf les plus pertinentes, ont été supprimées du poème afin d'éviter des contresens, des écarts de compréhension ou de fausses interprétations. Voici ce que les élèves ont proposé pour la première image :

La fin d'une journée d'orage,

Le soleil se couchant,

La suite des évènements situe le personnage clé du poème, le crapaud en le présentant sous plusieurs formes, l'image en question va de pair avec le ciel et l'étang, une forte description des lieux donnent une impression où se mêlent la nature et ses habitants. Les vers suivants, proposés par les élèves, illustrent cette description du paysage :

Un crapaud et/ou grenouille scrutait le ciel,

L'eau était claire et les oiseaux jouaient un air doux,

Sans aucune crainte, le crapaud contemplait le soleil,

Avec des yeux qui en disaient long,

\footnotetext{
${ }^{1}$ Il s'agit ici du texte intitulé « Le crapaud », extrait de La Légende des siècles, de Victor Hugo, paru dans le tome II L III, rédigé entre les 26-29 mai 1858 et publié en 1859, pp. 106-111.
} 


\section{Sur l’immensité du ciel}

La présentation des personnages à tour de rôle a mis les élèves dans une posture de curiosité allant du prêtre, à la femme et aux enfants. Chacun des personnages est présenté par les images qui reflètent, d'abord leur personnalité, ensuite les actes engagés sur ou avec le crapaud. Leurs propositions sont d'autant plus intéressantes, qu'elles associent l'imagination, la connaissance des propriétés de l'animal et une sorte de sincérité dans la perversité des personnages. Nous en présentons l'intégralité dans les vers suivants :

Un prêtre lui mis son talent sur le visage

Une jolie femme lui creva un œil

Quant aux enfants, ils durent s'efforcer pour faire souffrir l'animal,

Ils se moquent de sa nature hideuse,

Le crapaud souffrait affreusement,

Et les enfants le suivaient pour en finir avec lui.

Les images suivantes présentent un âne qui, fatigué, tire un charriot, il marche difficilement tant il est fatigué. Voici la rédaction toujours versifiée de cette partie :

Un âne fatigué, qui tirait un lourd charriot,

Abattue la bête marchait à peine,

Tant le poids de la journée lui faisait mal,

Son maitre hurlait sans cesse,

Le fouet du malheur ne laissait point de soupir à l'âne,

Là, les enfants s'arrêtèrent de faire du mal au crapaud,

Ils pensaient que l'âne allait tuer le crapaud

En voyant le crapaud dans un état lamentable,

L'âne s'arrêta et refusait d'avance malgré les menaces de son maitre,

Le crapaud ne fut pas écrasé par l'âne,

Qui laissa le misérable vivre,

L'âne reprit son chemin sous le coup de fouet,

Par la grâce de l'âne, le crapaud put vivre.

L'âne est plus clément que les humains

La bête a plus de bonté que tous les saints de la terre.

En fin de troisième séance, nous avons remis aux élèves l'intégralité du poème afin de confronter leurs propositions. Ce fut là l'occasion de mesurer leur compréhension du récit avec les connaissances mobilisées face à un texte littéraire présenté sous forme d'images. Cette séquence a également permis aux élèves de développer, d'une part, des habiletés rédactionnelles, d'autre part, des capacités de compréhension de ce type de texte, dans un contexte certes soumis à des moyens numériques particuliers. Le bilan de ces trois séances peut se résumer en termes d'acquis linguistiques et langagiers et de maitrise des interactions.

\section{Acquis linguistiques et langagiers}

En projetant des images muettes sur l'écran, nous avions pensé que les élèves pouvaient avoir des difficultés à proposer un codage linguistique correspondant à la réalité du texte. Or, à aucun moment, durant les trois séances, les élèves n'ont éprouvé de lassitude à faire progresser l'histoire qui les passionnait, tant le développement de la trame narrative leur présente une sorte de divertissement littéraire, au gré de la découverte des suites d'évènements.

À la facilité de l'interprétation des images s'adjoint le plaisir de l'écriture, qui se confirme par des vers pleinement acceptables. Les habiletés linguistiques et les capacités langagières 
augmentent et mettent en évidence l'implication des élèves face à cette série d'images avec laquelle ils tentent de construire une interprétation appropriée.

\section{Les inférences}

N'étant pas en mesure de décontextualiser le fonctionnement du texte, les élèves n'ont pas eu à mobiliser des inférences particulières pour pouvoir comprendre la progression narrative. Pourtant, les images positionnaient clairement le récit dans un contexte naturel, étayé par une description favorable à l'émergence de son sens.

Dans cette perspective, les inférences dégagées aboutissaient inéluctablement aux hypothèses émises par les élèves. La couleur des images laissait apparaitre d'emblée une rime possible, afin de restituer cette atmosphère qui règne souvent en littérature.

Le recours ou la traduction via la langue maternelle, en l'occurrence l'arabe algérien, n'a été d'aucune utilité puisque la construction du sens, à partir des images présentées, se laissait glisser par l'ensemble des éléments explicites ; leur interprétation directement en français ne pouvait donc être qu'évidente.

\section{L'efficience des images}

Nous avons admis que l'image est la représentation efficiente de l'œuvre littéraire. Dans ce sens, elle peut être partie intégrante de la description, de la narration et de la progression des évènements.

Les différentes approches adoptées par les élèves, afin d'aboutir à une compréhension de l'ensemble des images en mouvement, sont le témoignage d'une aptitude à générer du sens et de construire des significations cohérentes. Ils l'affirment dans la reconnaissance des images, en les distinguant les unes des autres de par leur dimension esthétique décomposée en différentes phases structurées, selon les besoins de la trame narrative.

Les différents personnages présentés tout au long du texte traduisent une cohésion inspirée par ces images. C'est ce qui renforce les idées mises en œuvre et rédigées par les élèves en interprétant les images. Les textes littéraires, pris dans ce contexte numérique, sont parfois portés par des images ou des suites d'images, à la façon d'une $\mathrm{BD}$, qui ne sont pas toujours accompagnées d'un codage linguistique particulier qui peut s'assimiler à son texte.

Sans chercher à faire l'apologie des textes numérisés et des supports imagés, nous constatons que cette approche des textes littéraires peut accroitre l'imagination des élèves et leur offrir des interactions facilement repérables; ces procédés et supports contribuent à former esthétiquement les esprits des élèves de langue étrangère.

\section{Maitrise des interactions}

F. Chacón (1992) a soutenu que l'ordinateur était une extension de trois comportements, appelés modes utilisateurs (user mode) :

- Le traitement de l'information correspondant à : j’apprends en faisant.

- L'interaction personne/machine : j’apprends par moi-même ou je suis enseigné.

- La communication personne/personne : j’apprends en interagissant avec d'autres.

Ces fonctions assurées par l'ordinateur sont légèrement différentes, mais surtout l'observation des réalisations montre qu'elles peuvent être interprétées ou mises en œuvre en fonction de visions éducatives complémentaires : 


\section{Participation au discours}

- Environnement de transmission de l'information où les élèves reçoivent de l'information.

- Environnement de communication où les élèves participent au discours en fixant leurs propres buts, en résolvant des problèmes, en créant du sens, en construisant de nouvelles informations et en fixant leurs propres critères de succès.

\section{Participation aux activités}

- Environnement de formation où les élèves réalisent des exercices afin d'améliorer leurs connaissances et leurs habiletés spécifiques.

- Environnement de lecture où les élèves travaillent sur des supports numérisés.

\section{Présentation de travaux aux fins d'évaluation}

- Environnement de test où les élèves démontrent leurs habiletés rédactionnelles et compréhensives afin de répondre à l'ensemble des questions.

- Environnement de performance où les élèves présentent leurs travaux à un public.

\section{Conclusion}

De manière plus fondamentale et par définition, les NTIC, en tant qu'outils au service d'actions éducatives et d'acquisitions langagières, peuvent s'accorder avec l'approche par compétences qui est au cœur de la réforme. Au-delà de ce contenu ou du sens qui peut être donné à l'introduction des NTIC dans le système éducatif algérien, les NTIC représentent, pour les décideurs et les acteurs du système éducatif, un moyen d'action et un levier pour la réalisation des objectifs de scolarisation, une opportunité de réussite face au défi que représente la réforme. La question essentielle pour réussir cette ambition n'est pas seulement technique ; les meilleures technologies sont aujourd'hui gratuites pour ceux qui savent participer équitablement aux larges communautés qui, dans le domaine de l'éducation comme ailleurs, contribuent à les mettre au point, à en partager les usages. Les questions clés sont bien celles de la compétence des professionnels de l'éducation et de leur formation qui permettront de s'approprier au mieux les outils qui évoluent sans cesse, et facilitent de plus en plus l'ensemble des activités éducatives, depuis la gestion jusqu'à la pédagogie. C'est donc avec l'intention de développer les compétences des acteurs du système éducatif algérien et de commencer à familiariser nos élèves avec ces nouveaux outils que nous leur donnerons l'occasion de mieux structurer leur formation.

L'introduction des moyens technologiques dans l'ensemble des structures éducatives est un tremplin pour le développement des connaissances des élèves, par la recherche constante d'autres horizons et d'autres perspectives. L'expérience menée restituée ici, dans le cadre de l'utilisation des NTIC pour l'enseignement du texte littéraire à l'ère du numérique, nous a permis de comprendre que les particularités du texte littéraire exigent un engagement intellectuel de l'auteur, et requiert en retour sa compréhension par le/les lecteurs. Avec l'utilisation des NTIC, cette complexité de compréhension s'est estompée car les images affichées rendent compte de la littéralité différemment des mots et du texte ; ce qui n'exclut pas, bien évidemment, la mobilisation des habilités langagières et linguistiques des élèves. La lecture hypermédia exige le développement de nouvelles compétences, non seulement parce qu'elle appelle la recherche d'informations dans différents modes sémiotiques (oral, écrit, images) et selon différentes modalités sensorielles (vision, ouie, toucher avec la souris), mais aussi parce que le mode d'organisation des documents se trouve diversifié par rapport à l’imprimé. 
Enfin, l'action didactique, face à ce type de texte numérisé, montre qu'il est plus facile de repérer les dysfonctionnements de compréhension des élèves, surtout si certaines notions narratives ou rédactionnelles sont difficilement maitrisables. Les relations qu'entretiennent les élèves avec les NTIC permettent de clarifier l'action pédagogique et le développement linguistique. Ce lien est mis en évidence dans la manifestation d'une compréhension efficiente et d'une herméneutique inhérente au texte littéraire.

La nécessité de motiver et d'amener les élèves de langue étrangère à dépasser l'attitude d'un consommateur passif consiste à leur assigner des objectifs didactiques précis. C'est le rôle et la fonction dévolus à toute institution éducative dans les années à venir.

\section{Abdelkader BENALI}

\section{Références bibliographiques}

ABDALLAH-PRETCEILLE, M. \& PORCHER, L. (1996). Éducation et communication interculturelle. Paris : Presses universitaires de France.

BESSE, H. (1982). Éléments pour une didactique des documents littéraires. In J. Peytard et al. (dir.), Littérature et classe de langue (pp. 13-34). Paris : Hatier.

CHACÓN, F. (1992). A Taxonomy of Computer Media in Distance Education. Open Learning, 7(1), 12-27.

COLLECTIF (2002). Y a-t-il un français sans objectif(s) spécifique(s). Les Cabiers de l'Asdifle, 14, Actes des 29 et 30èmes rencontres. Paris, mars 2002, Grenoble, octobre 2002.

COMPAGNON, A. (2007). La Littérature pour quoi faire? Leçon inaugurale au Collège de France. Paris : Fayard.

HUGO, V. (1859-1883). La Légende des siècles (extraits des tomes II L III « Le crapaud ») pp. 106111.

NIELSEN, J. \& LORANGER, H. (2006). Prioritizing Web Usability. Berkeley, CA : New Riders.

MAINGUENEAU, D. (1990). Pragmatisme pour le discours littéraire. Paris : Bordas.

MAINGUENEAU, D. (2003). Éléments de linguistique pour le texte littéraire. Paris : Nathan (4ème éd.).

MINISTÈRE DE L'ÉDUCATION NATIONALE (ALGÉRIE), direction de l'enseignement général (2000). Programmes de français langue étrangère : 1ère, 2ème et 3ème AS.

PERRENOUD, P. (2001). Dix nouvelles compétences pour un métier nouveau. In A.S. Lobo \& P. Feytor-Pinto (dir.), Professores de Português : Quem somos? Quem podemos ser? (pp. 47-54). Lisboa : Escola superior de Educaçao de Lisboa.

QEAU, P. (2010). Internet, révolution culturelle. Le Monde diplomatique, "Manière de voir », 109. THOMPSON, J.G. (2007). La Gestion de classe au secondaire. Guide pratique. Montréal : Chenelière éducation.

UNESCO - ONPS (2006). Réforme de l'éducation et innovation pédagogique en Algérie. 